Rabaska

Revue d'ethnologie de l'Amérique française

\title{
De la pertinence du musée
}

"Diriger sans s'excuser ".Patrimoine, musée et gouvernance selon Roland Arpin. Sous la direction d'Yves Bergeron et de Julie-Anne Côté. L'Harmattan, 2016, 333 p. ISBN 978-2-343-09523-3

Un nouveau musée pour un monde nouveau. Musée et muséologie selon Roland Arpin. Sous la direction d'Yves Bergeron et de Julie-Anne Côté. L'Harmattan, 2016, 342 p. ISBN 978-2-343-09524-0

\section{Michel Côté}

Volume 15, 2017

URI : https://id.erudit.org/iderudit/1041127ar

DOI : https://doi.org/10.7202/1041127ar

Aller au sommaire du numéro

Éditeur(s)

Société québécoise d'ethnologie

ISSN

1703-7433 (imprimé)

1916-7350 (numérique)

Découvrir la revue

Citer cette note

Côté, M. (2017). De la pertinence du musée / "Diriger sans s'excuser ".

Patrimoine, musée et gouvernance selon Roland Arpin. Sous la direction d'Yves Bergeron et de Julie-Anne Côté. L'Harmattan, 2016, 333 p.

ISBN 978-2-343-09523-3 / Un nouveau musée pour un monde nouveau. Musée et muséologie selon Roland Arpin. Sous la direction d'Yves Bergeron et de Julie-Anne Côté. L’Harmattan, 2016, 342 p. ISBN 978-2-343-09524-0. Rabaska, 15, 189-191. https://doi.org/10.7202/1041127ar d'utilisation que vous pouvez consulter en ligne. 


\section{De la pertinence du musée}

Michel CôTé

Muséologue

Les publications sous la responsabilité de Yves Bergeron et Julie-Anne Côté retraçant les réflexions de Monsieur Arpin sur la culture et la gestion ont le mérite, au-delà de conserver les traces d'une pensée agissante, de porter à notre attention des enjeux toujours actuels en matière de politiques et d'actions culturelles. Bien sûr, depuis la rédaction de ces textes, la société a évolué ; la grammaire muséographie s'est enrichie, des technologies se sont développées influençant l'expression et la pratique culturelle, des attentes se sont modifiées dans un monde de plus en plus ouvert, mais toujours divisé par de multiples inégalités... Pourtant, la pertinence des approches, l'analyse et la lecture des situations, tout comme les propositions d'actions demeurent des sources d'inspiration et d'opérationnalisation. Les spécialistes invités à commenter les propos de Monsieur Arpin confirment l'impact de la création du Musée de la civilisation sur le monde de la muséologie, sans tomber dans une naïve sacralisation de ce leader culturel. L'esprit critique de Monsieur Arpin commanderait d'ailleurs la nécessaire distanciation et la prudence dans l'analyse conjoncturelle.

Monsieur Arpin était donc un leader culturel. D'abord parce qu'il portait une vision. Il avait défini le Musée comme un espace thématique et pluridisciplinaire, un instrument pour tenter de comprendre et d'expliquer le monde. Pour ce faire, il n'était pas question de se restreindre à une seule discipline, mais de se nourrir d'un ensemble de grilles de lecture. Grand lecteur d'essais et consacrant beaucoup de temps lors des comités de direction aux débats de contenu, il se préoccupait d'aborder la complexité du réel en présentant nuances et points de vue. La programmation culturelle était élaborée en tenant compte des débats et enjeux de la société et en sachant que la société québécoise s'inscrivait dans des ensembles plus vastes. Son projet culturel était enraciné au Québec, mais ouvert sur le monde. Et ce, dans un esprit de rigueur scientifique. Monsieur Arpin se méfiait des idéologies et des partis pris. La crédibilité d'une institution muséale passe bien évidemment par 
cette expérience de la parole soutenue par des faits et la reconnaissance de nos ignorances. Il est intéressant de constater à rebours que le Musée se soit intéressé, dès son ouverture, aux enjeux liés à l'environnement, à la diversité culturelle, aux technologies, à la démographie, etc., enjeux qui continuent encore aujourd'hui à préoccuper les citoyens.

Monsieur Arpin était aussi un éducateur ; il venait du monde de l'enseignement, des cégeps et du ministère de l'Éducation (avant la Culture et le Trésor). Le Musée parle à qui, comment et pourquoi ? Il ne faut pas se surprendre de constater que le Musée de la civilisation ait développé une approche muséopédagogique importante, reconnu le rôle des guidesanimateurs, privilégié le développement d'un fort secteur culturel (cinéma, théâtre, littérature, danse, sciences, sciences humaines...) ou un service d'évaluation des publics. Ceux-ci constituent la base de l'action du Musée et ce, dans une double perspective : celle de la démocratie culturelle et celle de la fidélisation des visiteurs, donc de la durée. Le Musée est un service public et s'adresse à l'ensemble de la population; en conséquence, il doit développer une approche multicommunicationnelle, toucher à l'ensemble des savoirs et modes d'apprentissage. Les engagements envers des publics ciblés comme les analphabètes, les expositions nomades circulant dans divers milieux sur le territoire, les pratiques de partenariat avec les réseaux sociaux et culturels, les pratiques d'évaluation... tous les moyens étaient explorés pour faire du Musée non pas une institution que l'on visite, mais que l'on fréquente... Voilà sans doute une contribution remarquable à la muséologie; non pas parce que les institutions ne se préoccupaient pas d'éducation, mais en mettant au centre des actions la dimension communication/éducation. Monsieur Arpin donnait une nouvelle dimension au Musée, centrait son action sur la personne, sur l'échange, sur le visiteur. On ne peut imaginer aujourd'hui les débats suscités par cette lecture thématique, pluridisciplinaire, préoccupée par la place du visiteur actuel et celui à conquérir dans un souci de développement des connaissances et de réflexion.

Monsieur Arpin, les textes nous le confirment, était aussi un homme d'institution. Il croyait à la nécessité et à l'importance de celle-ci et avait un profond respect pour la dimension politique (et non politicienne) de la société. Il était aussi un homme d'action. Le véritable leader est d'ailleurs celui qui porte une vision, qui sait la partager et la transformer en réalisations. Au ministère de la Culture, il demandait de présenter les politiques sous forme de plans d'action obligeant de décrire mesures et programmes.

En parallèle à la publication du projet culturel du Musée de la civilisation, Monsieur Arpin faisait connaître les règles de fonctionnement et les stratégies opérationnelles. L'efficacité, l'efficience et l'impact se mesurent. Le Musée 
était structuré et les processus décrits (on ne peut imaginer le nombre d'activités nécessaires réalisées par une variété de métiers spécialisés pour produire une exposition). Une attention particulière était accordée à la gestion des ressources humaines. Tout en assumant son leadership, M. Arpin savait faire preuve d'écoute et de respect. On pouvait constater une grande adhésion au projet culturel ; sa présence a été palpable bien longtemps après son départ.

Il a su tirer profit et développer les compétences des membres de son équipe tout en maintenant le cap vers un objectif commun, encadrer en permettant de briser parfois le cadre pour mieux répondre aux attentes, donner le goût de participer à la réalisation d'un projet unique. Devenir un lieu de référence pour l'ensemble de la population exige de laisser une place à la remise en question et aux expérimentations, de s'ouvrir aux expertises et compétences internes et externes. Monsieur Arpin a toujours reconnu la contribution de son équipe et des collaborateurs/partenaires de l'extérieur. Le musée est perméable à son environnement et sait que sa réussite repose sur la compétence et l'engagement d'une foule d'individus et d'autres lieux et institutions.

Nous aurions pu analyser chacune des fonctions muséales ou les politiques culturelles élaborées, en mesurer les forces et faiblesses ; nous avons préféré, à la manière de Monsieur Arpin, nous en tenir aux fondamentaux, à ce qui constitue un apport et un enrichissement à la communauté, à ce qui se dessine de plus en plus comme des postulats nous permettant de reconnaître un leader culturel, celui qui porte une vision partagée et opérationnalisée. Monsieur Arpin n'est pas le seul; d'autres en musique, en danse ou au théâtre ont démontré une lecture cohérente de leur discipline ou un engagement thématique, social ou culturel et ont su transformer le projet culturel en produit et activité touchant et transformant des publics. En lisant des documents racontant leurs visions et démarches, nous ne pouvons que nous enrichir. Merci aux auteurs. 